\title{
Experiences with perinatal death among midwives, obstetricians, and assistant nurses: Results from a qualitative study in Norway Erfahrungen von Hebammen, Geburtshelfern und Kinderpflegern mit perinatalem Tod: Ergebnisse einer qualitativen Studie in Norwegen
}

\author{
Beate André1,2*, Raija Dahlø ${ }^{1}$, Tina Eilertsen ${ }^{3}$, \\ Shefaly Shorey ${ }^{4}$, Gerd I. Ringdal ${ }^{5}$ \\ ${ }^{1}$ Department of Public Health and Nursing, Norwegian \\ University of Science and Technology, Trondheim, Norway \\ ${ }^{2}$ NTNU Center for Health Promotion Research, Trondheim, \\ Norway \\ *beate.andre@ntnu.no \\ ${ }^{3}$ Clinic for surgery, Health Nord-Trøndelag, Levanger, \\ Norway \\ ${ }^{4}$ Yong Loo Lin School of Medicine, National University of \\ Singapore, Singapore \\ ${ }^{5}$ Department of Psychology, Faculty of Social Sciences and \\ Technology Management, NTNU, Trondheim, Norway
}

Received 19 February 2019, accepted 27 December 2019

\begin{abstract}
This study aims to explore experiences of healthcare professionals, including midwives, obstetricians, and assistant nurses, after a perinatal death. A qualitative study design was used, and 20 participants were interviewed. Data were analyzed using content analysis. Three categories emerged from the data: (i) external responses, (ii) internal responses, and (iii) personal responses. Generally, there was a "culture of blame" in response to the perinatal death among the professionals. The lack of opportunity to deal with perinatal death and having an inappropriate way to handle feelings and experiences are the important findings of this study. If not well supported, the concerned healthcare professionals may face serious health challenges in the long term. Hence, supportive interventions need to be designed and evaluated to provide adequate support to the concerned professionals.
\end{abstract}

\begin{abstract}
Diese Studie untersucht die Erfahrungen Gesundheitsfachkräften (Hebammen, Gynäkologen, Hilfspflegerinnen) nach einem perinatalen Tod zu untersuchen. Es wurde ein qualitatives Studiendesign verwendet und 20 Teilnehmer/-innen wurden befragt. Die Daten wurden mittels Inhaltsanalyse analysiert. Aus den Daten gingen drei Kategorien hervor: i) externe Antworten, ii) interne Antworten und iii) persönliche Antworten. Im Allgemeinen gab es eine „Kultur der Schuld“ als Reaktion auf den perinatalen Tod unter den Fachleuten. Die wichtigsten Ergebnisse dieser Studie sind die mangelnde Möglichkeit, mit dem perinatalen Tod umzugehen und eine unangemessene Art und Weise, mit Gefühlen und Erfahrungen umzugehen. Wenn sie nicht gut unterstützt werden, könnten die betroffenen Angehörigen des Gesundheitspersonals langfristig ernsthaften gesundheitlichen Problemen ausgesetzt sein. Daher müssen unterstützende Maßnahmen konzipiert und bewertet werden, um die betroffenen Fachleute angemessen zu unterstützen.
\end{abstract}

\section{Keywords}

Perinatal death - self-blame - guilt - contrasts

\section{Keywords}

Perinataler Tod - Selbstbeschuldigung - Schuld - Kontraste

\section{BACKGROUND}

Midwives, obstetricians, and the assistant nurses, as well as other healthcare professions, are exposed to stress at work (Borritz et al., 2006). Critical events may lead to extreme stress for healthcare professionals (de Boer, van Rikxoort, Bakker, \& Smit, 2014). Perinatal death is defined as a stillbirth occurring at $\geq 22$ completed weeks of gestation, a death during birth, or a death during the first week of life (Zegers-Hochschild et al., 2009). 
Encountering perinatal death has a severe influence on healthcare personnel. Common reactions are worry, strain, blame, and self-blame (Andre, Dahl $\varnothing$, Eilertsen, \& Ringdal, 2016). The absence of acknowledging the responses among midwives, obstetricians, and nurses appears to be most provoking and can cause suppression of emotions. This may further lead to unprocessed sad experiences and low self-confidence among concerned professionals (McCreight, 2005; Walker, Mashiach, \& Manny, 2016). Withdrawal and rejection were shared responses to perinatal death among healthcare professionals. These responses may further influence the care and support they offer to the grieving relatives (Andre et al., 2016). Previous literature have highlighted the need of supporting healthcare professionals by providing them training in communication with grieving relatives and support from supervisors to handle their responses in a more successful manner (Andre et al., 2016; B. André, Frigstad, S.A., Nøst, T.H., Sjøvold, E, 2016).

The communication of feelings by the healthcare professionals needs to be implanted in the work culture and as an element of specialized training for nurses (Andre et al., 2016; Shorey, André, \& Lopez, 2016). However, in acknowledging emotional responses, there seems to be a dissimilarity among nurses and midwives as well as doctors and obstetricians. Although nurses found it normal to acknowledge and share their feelings, the doctors, on the other hand, found it undesirable to show and share their emotions (Farrow, Goldenberg, Fretts, \& Schulkin, 2013). Previous literature have shown that those healthcare professionals who do not share or acknowledge their emotional distress after perinatal death tend to leave the organization, suffer with psychological distress, and have feelings of burnout (Gold, Kuznia, \& Hayward, 2008; McCool, Guidera, Stenson, \& Dauphinee, 2009). Although midwives were found to acknowledge and share their emotional distress, lack of organizational support made them feel burnout and they consider leaving the profession (Shorey et al., 2016). Even if different professions seem to have different approaches and experiences to the perinatal death, the burden they experience in these situations can be equated. Other studies found no variances between the professions in their understanding of the emotional consequences associated with perinatal death. The feelings of pressure, sadness, blame, grief, and mourning are the shared feelings exhibited by midwives, obstetricians, and nurses (Farrow et al., 2013; Gold et al., 2008; Jones \& Smythe, 2015; McCool et al., 2009; Nallen, 2007; Pastor Montero et al., 2011; Wallbank, 2010). The need of individualized, cultural-specific informational support and implementing such supportive interventions right from the professionals training schools in their curriculums have been recommended (Ben-Ezra M, 2014; Steen, 2015; Walker et al., 2016). Reactions from healthcare personnel meeting perinatal death in clinical practice are encircled by "a conspiracy of silence" and "denial within the profession" (Cowan \& Wainwright, 2001; Mander, 2009; Redinbaugh et al., 2003). These responses are not only distressing for healthcare personnel who experience this avoidance behavior but can also affect care given to parents (Cowan \& Wainwright, 2001).

Research has shown that healthcare professionals working in maternity units exhibit moderate secondary stress (Beck \& Gable, 2012) and midwives have experienced clinical post-traumatic stress disorder, which is further associated with burnout (Sheen, Spiby, \& Slade, 2015). Doctors who cared for parents with perinatal death experienced considerable emotional trauma, and some of them considered abandoning their obstetric practice (Gold et al., 2008). Seeing the importance of healthcare professionals' experiences and needs in caring for bereaved parents, recent reviews have focused on the perspectives of healthcare professionals working in maternity units (Ellis et al., 2016; Wallbank \& Robertson, 2008). As culture influences expressions and attitudes about grief, it is important to examine the experiences and needs of healthcare professionals from diverse cultures (Gibson, 2006; Shorey et al., 2016).

Given this background, the following research question will be explored:

As such this study aimed to explore the experiences of Norwegian healthcare professionals, including midwives, obstetricians, and assistant nurses, after experiencing perinatal death related to

- How do the leadership at the hospitals support healthcare professionals in processing their emotions and reactions?

- How do healthcare professionals process their emotions and reactions?

- How do these experiences affect healthcare professional's private life?

\section{METHOD}

A descriptive qualitative study design was used. We chose this design because there are few studies on this topic (Malterud, 2001) and because this is a sensitive topic. A semi-structured interview guide was used to interview the participants. Data collection took place from October 2016 to December 2016 at two public hospitals located at the central Norway. The data were collected in Norwegian and translated into English.

\section{Sample}

All healthcare professionals who have experienced the perinatal death in their career were invited to participate in the study. The inclusion criteria were (i) the healthcare 
Table 1: Examples of meaning units, condensation, categories, and coding.

\begin{tabular}{|c|c|c|c|}
\hline Meaning units & Condensation & Categories & coding \\
\hline $\begin{array}{l}\text { It is challenging to be in these situations- } \\
\text { so then we went together to the delivery } \\
\text { room. Having security in colleagues, it is } \\
\text { very important, no matter what the situa- } \\
\text { tions are. It did not end well, but it became } \\
\text { as good as it possible could be. }\end{array}$ & $\begin{array}{c}\text { Feeling safe among col- } \\
\text { leagues is important }\end{array}$ & External reactions & Leadership management issues \\
\hline $\begin{array}{c}\text { When the mother comes to the maternity } \\
\text { unit, they have experienced no fetal move- } \\
\text { ment. They have taken ultrasound to check } \\
\text { it and found minus fetal sound. You may } \\
\text { then have some time to prepare. There may } \\
\text { not be so many minutes to prepare, but } \\
\text { some. }\end{array}$ & $\begin{array}{l}\text { The mother comes in without } \\
\text { fetal sounds; the midwife has } \\
\text { some time to prepare }\end{array}$ & External reactions & Work situation \\
\hline
\end{tabular}

professionals should have at least two perinatal death experience and (ii) they should be able to read and speak Norwegian language. Twenty healthcare professionals participated in this study.

\section{Qualitative Research Interview}

The results consisted of in-depth interviews that continued from 40 to 50 minutes conducted for a limited time of 2 months. All participants were ensured that all data would be anonymous and that their participation was important to shed light on a little-explored area. All the interviews were audio recorded and transcribed verbatim. A semistructured interview guide was used, and all participants were encouraged to discuss their concerns freely (Miles, Huberman, \& Saldana, 2013). The interview guide was based on earlier studies (Andre et al., 2016; Cowan \& Wainwright, 2001; de Boer et al., 2014; Farrow et al., 2013; Gold et al., 2008; McCool et al., 2009; McCreight, 2005; Pastor Montero et al., 2011; Shorey et al., 2016; Wallbank \& Robertson, 2008) and organized in themes, such as experience, support, and emotional impact.

\section{Analyses}

Data were analyzed in five steps based on approaches and guidelines stipulated by Brinkmann and Kvale (2015) as follows:

1) Understand: at this step, the material was read repeatedly, and it was focused on both meaning and context. Different interpretations of the text in relation to meaning and context were discussed among the researchers.

2) Structure: at this step, the researchers read through the transcribed text several times and the meaning units were color-coded independently for each interview.

3) Summarize: at this step, we eliminated the superfluous material such as digressions and repetitions and made a distinguishing between the essential and nonessential text.

4) Create meaning: at this step, meaning condensation, meaning categorization, narrative structuring and meaning interpretation were performed as described below. The researchers made a deeper and theoretical interpretation of the text, for example, by creating categories and subcategories.

5) Meaning generation: at this step, the researchers merged the data and selected text to the categories and subcategories.

Two researchers participated in the data analysis independently. The two researchers derived the codes and categories independently and discussed between themselves until the consensus were made. Any discrepancy was discussed with the third researcher. Being a qualitative researcher, it is important to be aware of the significance of reflecting about one's part in the process of collecting, analyzing, and interpreting the data and pre-conceived expectations one bring to the study (Brinkmann, 2015; Korstjens \& Moser, 2018). Rigor was maintained by ensuring dependability and confirmability. Dependability comprises consistency in the presentation of findings using participants' quotes. Confirmability refers to the context of neutrality and intersubjectivity of the data. The concept of intersubjectivity refers to openness about the conditions under which knowledge is developed (Malterud, 2001; Stige, Malterud, \& Midtgarden, 2009). We ensured that interpretation of findings emerged from the participants' sharing and not from the researchers' own perspectives (Korstjens \& Moser, 2018).

\section{Ethical Considerations}

The ethical guidelines of voluntary participation, informed consent, and the possibility of withdrawal at any time were followed. Participants were informed about the purpose and aim of the study. All gathered data 
Table 2: Overview of categories and subcategories.

\begin{tabular}{l|c|c|c|}
\hline Categories & External Responses & Internal Responses & Personal Responses \\
\hline \multirow{2}{*}{ Subcategories } & 1) Leadership issues & 1) Self-blame & 1) Personal reactions \\
& 2) Work situation & 2) Guilt & 2) Privacy \\
\hline
\end{tabular}

were anonymized. The Regional Committees for Medical and Health Research Ethics in Norway (2015/1138) reviewed the study. To maintain the confidentiality of the interviews, we will not report the results according to professional groups because we only have few participants in each group. For the same reason, we do not distinguish between genders.

\section{RESULTS}

The findings are organized in three categories with subcategories that emerged from the data. These categories are: (i) external responses, (ii) internal responses, and (iii) personal responses. Healthcare professionals commented on external responses, when issues outside their own control or influence, that prejudiced on their experiences with perinatal death. Internal responses are comments that label issues related to their performance as a professional and how they describe their feelings in these situations. Personal responses are comments concerning themselves as a person and the affect these experiences have on their personal life. Categories and subcategories are presented in Table 2. The participants were consecutively numbered, and these numbers have been put in parentheses at the end of each statement.

\section{External responses}

This category consists of the subcategories including leadership issues, work situation, and system issues.

\section{Leadership issues}

Many participants described being blamed for the incident. A lack of support and understanding from both leaders and colleagues were expressed. Having support was highlighted to be vital for successful processing of one's thoughts.

Someone was blamed for not having a professional background. This has been disappointing. One must feel safe. We must support each other. (11)

Feeling safe among colleagues is important. (1)
Participants indicated that looking for someone to blame was very stressful. Being in situations with perinatal death was demanding, and the experience of being pointed out as guilty of what has happened makes it even worse. They highlighted that the leadership should take responsibility to foster a culture of no blame.

Experiencing being blamed where you know you are innocent-Insecurity next time. (12)

Feeling unsafe, still "foggy," characterized by the incident. (11)

Leadership's responsibility to make sure that the healthcare personnel can process their feelings was emphasized. Many felt this was not taken seriously and that routines for this were not provided at the unit.

It is painful not to be "seen" by the leader or asked how it is going; it gives the feeling of not being interested. (20)

Leaders never ask how you feel inside or if you are okay. (16)

Supervisors should be responsible and ask, 'Has anything happened this weekend that must be discussed?' (1)

\section{Work situation}

Participants stated that just having some time to prepare is positive and may contribute to better care of the parents. At the same time, often situations with perinatal death occur acutely and unexpectedly, so it is not possible to prepare in advance.

The mother comes in without fetal sounds; the midwife has some time to prepare. (5)

You can prepare better if you know beforehand that a birth occurs with cessation of fetal sounds. (2) 
Generally, there is much happiness in a maternity ward, so the contrast with perinatal death is, therefore, particularly high.

Anti-climax on a maternity unit; it is sad with a big contrast. (8)

It is completely on the other side compared to the way it should be, a very big contrast. (2)

Some participants described being simultaneously responsible for normal births and perinatal death as especially challenging. Experiencing such contrasts is demanding because there will be a meeting of both joy and sorrow and where to be involved in both situations. It is difficult to help parents in terms of both joy and sadness.

It is overwhelming to walk between sadness and joy. (7)

It is hard to "wear the mask" when walking between stillbirths and "common" births. (16)

\section{System issues}

The following statements describe how this incidence especially was experienced as difficult because the system does not facilitate good enough. There should have been procedures to allow healthcare personnel to process their emotions.

I had to keep working instead of getting some free time afterward. I had to try to proceed as if nothing had happened. (11)

Anyone who is vulnerable should be given the opportunity to process their feelings before going back to work. (13)

I should have been allowed to go home afterward.

\section{Internal responses}

This category consists of the subcategories such as guilt and self-blame. Participants felt that they should have provided more answers or done something to prevent perinatal death. It is natural for healthcare professionals to feel guilty and blame themselves; expectations of healthcare are high today. When a child dies, one can feel that they have not met these expectations.

\section{Guilt}

The participants described feelings of guilt. They expressed fear of having done something wrong.

It took time before you realize that this was not something you could have expected. (13)

It feels many times that I should have done things differently. Trying to think...I did as well as I could. I cannot be afraid to go to work. (10)

It is easy to blame yourself. Have I done something wrong? Have I done well enough? (7)

You feel guilty when the child dies during or after birth. (20)

It is worse for us when the child dies in the hospital. You feel responsible whether you could have done anything to prevent it or not. (10)

It can be difficult to accept that a small child has died. One informant also had thoughts about "fair" and "unfair" in a perinatal death situation.

Do not think about what is fair or unfair. There is no answer to what is fair or unfair. (12)

Several participants had thoughts about the seriousness and their responsibilities in these situations. Situations with perinatal death can quickly develop into a critical situation, and healthcare professionals must take rapid decisions.

The sense of responsibility is prominent. Decisions must come immediately. There is not an opportunity to read a guidebook. There is not much room for mistakes. (11)

This is exactly what we are afraid of - making a bad assessment that has tragic consequences. (15)

I tell myself there was nothing I could have done differently. You must have some methods of survival, and you must put things behind you. (5)

Some described a situation in which a child dies in the hospital and they cannot determine the cause of the death. If one finds the cause of death of the child, it may be easier to give the parents an explanation and easier for healthcare personnel to process the event. 
It is easier to reconcile if one finds a cause-important also for health professionals-common with guilt whether cause of death is found. (5)

\section{Self-blame}

Many participants described feelings of self-blame regarding what happened. Self-blame is a natural feeling that can occur in such situations, and this is a significant burden on the health worker.

Feeling guilty and blaming oneself on behalf of the system or other healthcare professionals who have not discovered a worrying situation earlier. (15)

It indicates that the child has no heartbeat. The time just stops-it is a terrible situation. You feel selfblame. (14)

We have come to terms with the fact that we do not accept things that are not perfect and controllablequestions arise about guilt, fault, and self-blame. (2)

\section{Personal responses}

This category consists of the subcategories such as personal reactions and privacy. Participants also stated how the situations with perinatal death influenced on their personal life and their perception of themselves as a professional or a human.

\section{Personal reactions}

Health professionals experience both good and bad working days similar to others. Some days they did not feel good and the other days were better. Coping with situations of perinatal death require them to be at their best.

I do not volunteer immediately. We try to distribute such incidents. (5)

You try to avoid the situation if you are not feeling completely at the top. (11)

If experiencing sadness and loss or having problems in private life, you are more vulnerable in such situations. (5)

The participants described their paths and memories in different ways. Perinatal death is a watershed moment and, therefore, has a serious impact on those involved.
I have been thinking a lot about the situation, when it occurred and what happened afterward. (18)

It stays in your head for a while. It does not go away for a while. (12)

I remember and think about the incident occasionally. (14)

Some participants expressed the challenge of dealing with their feelings while simultaneously dealing with the parents' feelings. It is hard to know how much they should show their personal feelings in these situations.

It is challenging to deal with your own feelings. (4)

It is challenging with the balance between your own feelings and professionalism. (6)

I was a little taken by surprise, because it is not easy to predict your own reactions. (3)

Experience with these situations can make one more confident or just the opposite. Being involved does something to healthcare professionals, and emotions need to be processed to move on from the experience.

The first experience was the most difficult because you had to deal with your own reactions along the way. (4)

Experience leads to setting aside your own feelings, and you manage to give of yourself to the parents. (6)

Eventually, I became less sensitive. I learned not to show feelings to parents. You must be a strong person who can support and be trusted. (9)

Some reactions described by participants are examples of unhealthy approaches to processing feelings. These approaches can lead to avoidance and emotional trauma.

I now handle a burdensome situation well. Being professional primarily, you do the best you can. Do not show feelings for the patient. (12)

Shut off all feelings and images so they do not stand in the way of what you need to do to perform your job. (3)

Try to think of something else if emotions are overwhelming. (4) 


\section{Privacy}

The participants expressed in many ways how perinatal death influenced their private lives. The fact that one's privacy is affected by situations encountered in the workplace is serious and can lead to a deterioration of the quality of life for those involved.

Post-traumatic stress symptoms - I experience sleep loss and feel that my family is suffering. (11)

We go home to our families. We have children we come home to. You have your thoughts. (17)

\section{I experienced sleep loss and weight loss. (13)}

The participants described their experiences with perinatal death in different ways, but everyone sees them as particularly traumatic events. They described the impact of the situation on their personal lives.

I experience being disappointed with myself. (11)

It is a traumatic situation, and it gets stuck. I remember everything very clearly, really detailedvery emotional in a negative way. (14)

You do not forget. We are not robots. (19)

\section{DISCUSSION}

The aim of this study was to explore the experiences of midwives, obstetricians, and assistant nurses pertaining to perinatal death. Earlier studies showed that there is little or insignificant difference in the emotional experience after experiencing perinatal death among different professional groups (Andre et al., 2016; McCreight, 2005; Rich, 2002). Therefore, in this study, all the professional groups were considered as one inter-professional group during the data collection, analysis, and reporting of the findings.

The participants defined many external factors that influence their experiences after experiencing a perinatal death. They described that they were blamed for what happened without having done anything wrong. This may be described as a "culture of blame" and looking for blame (Andre et al., 2016). These findings are consistent with previous studies that reported fostering a no-blame culture is management's responsibility (Nallen, 2006). Other researchers also stated that this is an issue that management must face (Jones \& Smythe, 2015; McCool et al., 2009; McCreight, 2005; Nallen, 2007). But even having this knowledge, our findings suggest that the leadership at maternity units failed to meet this responsibility. Feeling safe and secure in their professional practice is important for professionals to deal with this challenging situation in the best way. Lack of support may be very challenging for the healthcare personnel at these units because of overall feeling of guilt and selfblame. Some participants also stated that the supervisors or leaders did not ask about them or discuss their feelings associated with perinatal death. This may lead to serious consequences for the concerned professionals. The leadership need to investigate developing a supportive system for the healthcare professionals facing perinatal death (Andre et al., 2016). The participants characterized these situations as traumatic and very emotional, and some of them stated that their feelings "get stuck." However, if healthcare personnel process these experiences in a better way, they may feel less traumatized and become a greater resource for the bereaved parents (Andre et al., 2016; Shorey et al., 2016). If things such as better support system and knowledge enable healthcare personnel to support the affected parents more appropriately, then this must be developed to foster higher competence. Some participants explained that their experiences led them to "become less sensitive" or "not to show feelings when they are with the parents" or "shut off all feelings" or "try to think of something else." These statements from the participants describe ways of handling the situation without processing their own emotions. Responses such as this to significant reactions are not just troubling for staff subject to the negation but could also be a challenge for them when giving the parents the best possible treatment (Cowan \& Wainwright, 2001).

Many participants also described the contrast of delivering healthy babies in one situation and perinatal death in another as challenging. As stated by participants, it is hard to "wear a mask" when walking between stillbirths and "common" births. This is difficult for the management as well because these situations often occur suddenly and without time to prepare. It seems important, however, for healthcare personnel to be able to concentrate on the perinatal death without having to participate in ordinary births at the same time (André, 2000; Andre et al., 2016; Nallen, 2006, 2007). Concentrating on the perinatal death alone may also be essential in allowing healthcare personnel to have the time to process the experience in a constructive way and may make it easier for them to reflect on the situation (Kahan \& Goodstadt, 2001).

When describing internal responses to perinatal death, healthcare personnel described that they felt guilty and blame themselves, even if there was no valid reason to do so. The participants stated that they felt responsible whether they could have done anything to prevent the death, or not. The feeling of responsibility and the seriousness of the situation make healthcare professionals think carefully about what they have done and whether 
they could have done something differently. Almost all participants talked about guilt and self-blame in some form. It seems natural that this feeling will occur in these situations, and healthcare personnel who work in maternity units should be given knowledge about such circumstances (B. André, 2000; Farrow et al., 2013; Jones \& Smythe, 2015; McCool et al., 2009; Nallen, 2006, 2007; Pastor Montero et al., 2011). If one knows that these are common feelings, it may be easier to deal with them (McCreight, 2005; Wallbank, 2010). However, the participants also described other feelings such as sadness and shock. Healthcare personnel must deal with their feelings while simultaneously meeting with the parents in their grief.

One of the most serious implications of not processing the experience in a constructive way is the impact on the healthcare personnel's private life. Some participants claimed to be experiencing "sleep loss and weight loss." The participants described post-traumatic stress symptoms and indicated that they had considered leaving the profession because of these events. The participants described difficulty in expressing their feelings. Earlier research has shown that healthcare personnel in maternity units often undergo negative impacts on their psychological well-being; they felt depressed and burned out (Ben-Ezra M, 2014; Shorey et al., 2016; Walker et al., 2016). Other studies have found that post-traumatic stress symptoms were present in healthcare personnel caring for parents with perinatal death (Sheen et al., 2015).

This study has shown that the participants described limited ability to process their emotions constructively. It seems imperative that programs must be implemented (Gold et al., 2008; McCreight, 2005; Wallbank, 2010) to focus on the emotional well-being of healthcare professionals and setbacks that can naturally occur in such positions. The benefit of sharing thoughts and emotions with each other is important knowledge for healthcare personnel who will be involved in such situations (McCreight, 2005; Rich, 2002).

\section{Limitations and strengths}

The dependability of this research, associated with the qualitative results, could be restricted as the data were collected from only two clinics. However, the two units are comparable in size and location, and we interviewed a reasonable number of participants. Because we wanted to ensure full anonymity, it was not possible to provide detailed information and differences among occupational groups and gender. Data were collected in Norwegian and it may be that some of the meaning of the content could have been lost during the translation of transcripts into English. Furthermore, the study has been carried out in a field where this focus has scarcely been described previously.

\section{CONCLUSION}

This study focused on factors that characterize the experiences of midwives, obstetricians, and assistant nurses when children died in connection with childbirth at two Norwegian hospitals. The contrast between perinatal death and "normal births" and the lack of time to prepare themselves to take care of bereaved parents in the best way were a significant finding in this study. Healthcare professionals' personal feelings in these situations, such as guilt, self-blame, sadness, and insecurity, were hardley given attention by the leaders. However, the lack of opportunity to deal with these situations and inappropriate ways to handle their feelings and experiences are important findings in this study. This may lead to serious health challenges in the long term for the concerned healthcare professionals. Future research about support specifically needed for healthcare providers experiencing perinatal death is needed.

\section{References}

André, B. (2000). Når møtet med livet blir møte med døden. . Vard i Norden, 20(2), 39-43.

Andre, B., Dahlø, R., Eilertsen, T., \& Ringdal, G. I. (2016). Culture of silence: Midwives', obstetricians' and nurses' experiences with perinatal death. Clinical Nursing Studies, 4(4), p58.

André, B., Frigstad, S.A., Nøst, T.H., Sjøvold, E. (2016). Differences in communication within the nursing group and with other professions at a hospital unit. . Journal of Clinical Nursing., Accepted article.
Beck, C. T., \& Gable, R. K. (2012). A mixed methods study of secondary traumatic stress in labor and delivery nurses. Journal of Obstetric, Gynecologic, and Neonatal Nursing, 41(6), 747-760.

Ben-Ezra M, P. Y., Walker R, Many A, Hamam-Raz Y. (2014). The impact of perinatal death on obstetrics nurses: a longitudinal and cross-sectional examination. $J$ Perinat Med., 42(1), 75-81. doi:10.1515/.jpm-2013-0071

Borritz, M., Rugulies, R., Bjorner, J. B., Villadsen, E., Mikkelsen, O. A., \& Kristensen, T. S. (2006). Burnout among employees in 
human service work: design and baseline findings of the PUMA study. Scandinavian Journal of Public Health, 34(1), 49-58.

Brinkmann, S. K., S. (2015). InterViews: Learning the Craft of Qualitative Research Interviewing (third edition ed.): sage Publications Inc.

Cowan, L., \& Wainwright, M. (2001). The death of a baby in our care: the impact on the midwife. Midirs midwifery digest, 11(3), 313-316.

de Boer, J., van Rikxoort, S., Bakker, A. B., \& Smit, B. J. (2014). Critical incidents among intensive care unit nurses and their need for support: explorative interviews. Nursing in Critical Care, 19(4), 166-174.

Ellis, A., Chebsey, C., Storey, C., Bradley, S., Jackson, S., Flenady, V., . . . Siassakos, D. (2016). Systematic review to understand and improve care after stillbirth: a review of parents' and healthcare professionals' experiences. BMC pregnancy and childbirth, 16(1), 16.

Farrow, V. A., Goldenberg, R. L., Fretts, R., \& Schulkin, J. (2013). Psychological impact of stillbirths on obstetricians. The Journal of Maternal-Fetal \& Neonatal Medicine, 26(8), 748-752.

Gibson, M. (2006). Order from chaos: Responding to traumatic events: Policy Press.

Gold, K. J., Kuznia, A. L., \& Hayward, R. A. (2008). How physicians cope with stillbirth or neonatal death: a national survey of obstetricians. Obstetrics and Gynecology, 112(1), 29-34.

Jones, K., \& Smythe, L. (2015). The impact on midwives of their first stillbirth. New Zealand College of Midwives Journal(15), 17-22. doi:https://www.midwife.org.nz/resources-events/nzcom-journal/ issue-51/the-impact-on-midwives-of-their-first-stillbirth/

Kahan, B., \& Goodstadt, M. (2001). The interactive domain model of best practices in health promotion: Developing and implementing a best practices approach to health promotion. Health Promotion Practice, 2(1), 43-67.

Korstjens, I., \& Moser, A. (2018). Series: Practical guidance to qualitative research. Part 4: Trustworthiness and publishing. European Journal of General Practice, 24(1), 120-124.

Malterud, K. (2001). Qualitative research: standards, challenges, and guidelines. The lancet, 358(9280), 483-488.

Mander, R. (2009). Good grief: Staff responses to childbearing loss. Nurse Education Today, 29(1), 117-123.

McCool, W., Guidera, M., Stenson, M., \& Dauphinee, L. (2009). The pain that binds us: midwives' experiences of loss and adverse outcomes around the world. Health Care for Women International, 30(11), 1003-1013.

McCreight, B. S. (2005). Perinatal grief and emotional labour: a study of nurses' experiences in gynae wards. International Journal of Nursing Studies, 42(4), 439-448.

Miles, M. B., Huberman, A. M., \& Saldana, J. (2013). Qualitative data analysis: Sage.

Nallen, K. (2006). Midwives' needs in relation to the provision of bereavement support to parents affected by perinatal death. Part one. Midirs midwifery digest, 16(4), 537-542.

Nallen, K. (2007). Midwives' needs in relation to the provision of bereavement support to parents affected by perinatal death. Part two. Midirs midwifery digest, 17(1), 105-112.
Pastor Montero, S. M., Romero Sanchez, J. M., Hueso Montoro, C., Lillo Crespo, M., Vacas Jaen, A. G., \& Rodriguez Tirado, M. B. (2011). Experiences with perinatal loss from the health professionals' perspective. Revista Latino-Americana de Enfermagem, 19(6), 1405-1412.

Redinbaugh, E. M., Sullivan, A. M., Block, S. D., Gadmer, N. M., Lakoma, M., Mitchell, A. M., . . Arnold, R. M. (2003). Doctors' emotional reactions to recent death of a patient: cross sectional study of hospital doctors. BMJ, 327(7408), 185.

Rich, S. (2002). Caregiver grief: Taking care of ourselves and our patients. International Journal of Trauma Nursing, 8(1), 2428. Retrieved from http://www.sciencedirect.com/science/ article/pii/S107542100286878X. doi:http://dx.doi.org/10.1067/ mtn.2002.121332

Sheen, K., Spiby, H., \& Slade, P. (2015). Exposure to traumatic perinatal experiences and posttraumatic stress symptoms in midwives: prevalence and association with burnout. International Journal of Nursing Studies, 52(2), 578-587.

Shorey, S., André, B., \& Lopez, V. (2016). The experiences and needs of healthcare professionals facing perinatal death: A scoping review. International Journal of Nursing Studies.

Steen, S. E. (2015). Perinatal death: bereavement interventions used by US and Spanish nurses and midwives. International journal of palliative nursing, 21(2), 79-86.

Stige, B., Malterud, K., \& Midtgarden, T. (2009). Toward an agenda for evaluation of qualitative research. Qualitative Health Research, 19(10), 1504-1516.

Walker, R., Mashiach, R., \& Manny, A. (2016). Comorbidity of posttraumatic stress symptoms and depressive symptoms among obstetric nurses with perinatal death exposure. The Israel journal of psychiatry and related sciences, 53(2), 58.

Wallbank, S. (2010). Effectiveness of individual clinical supervision for midwives and doctors in stress reduction: findings from a pilot study. Evidence-based Midwifery, 8(2), 65-71.

Wallbank, S., \& Robertson, N. (2008). Midwife and nurse responses to miscarriage, stillbirth and neonatal death: A critical review of qualitative research. Evidence-based Midwifery, 6(3), 100-107.

Zegers-Hochschild, F., Adamson, G. D., de Mouzon, J., Ishihara, O., Mansour, R., Nygren, K., . . V Van der Poel, S. (2009). The international committee for monitoring assisted reproductive technology (ICMART) and the world health organization (WHO) revised glossary on ART terminology, 2009. Human Reproduction, 24(11), 2683-2687. 